\title{
Involvement of angiotensin II in the remodeling induced by a chronic decrease in blood flow in rat mesenteric resistance arteries
}

\author{
Céline Baron-Menguy ${ }^{1,2,3}$, Bertrand Toutain ${ }^{1,2,3}$, Maud Cousin ${ }^{4}$, Odile Dumont ${ }^{1,2,3}$, Anne-Laure Guihot ${ }^{1,2,3}$, \\ Emilie Vessières ${ }^{1}$, Jean-François Subra ${ }^{3,4,5}$, Marc-Antoine Custaud ${ }^{1,2,3}$, Laurent Loufrani ${ }^{1,2,3}$ \\ and Daniel Henrion ${ }^{1,2,3,4}$
}

Blood flow reduction induces inward remodeling of resistance arteries (RAs). This remodeling occurs in ischemic diseases, diabetes and hypertension. Nonetheless, the effect of flow reduction per se, independent of the effect of pressure or metabolic influences, is not well understood in RA. As angiotensin II is involved in the response to flow in RA, we hypothesized that angiotensin II may also be involved in the remodeling induced by a chronic flow reduction. We analyzed the effect of angiotensin I-converting enzyme inhibition (perindopril) and angiotensin II type 1 receptor blockade (candesartan) on inward remodeling induced by blood flow reduction in vivo in rat mesenteric RAs (low flow (LF) arteries). After 1 week, diameter reduction in LF arteries was associated with reduced endothelium-dependent relaxation and lower levels of eNOS expression. Superoxide production and extracellular signal-regulated kinases 1/2 (ERK1/2 phosphorylation were higher in LF than in normal flow arteries. Nevertheless, the absence of eNOS or superoxide level reduction (tempol or apocynin) did not prevent LF remodeling. Perindopril and candesartan prevented inward remodeling in LF arteries. Contractility to angiotensin II was reduced in LF vessels by perindopril, candesartan and the ERK1/2 blocker PD98059. ERK1/2 activation (ratio phospho-ERK/ERK) was higher in LF arteries, and this activation was prevented by perindopril and candesartan. ERK1/2 inhibition in vivo (U0126) prevented LF-induced diameter reduction. Thus, inward remodeling because of blood flow reduction in mesenteric RA depends on unopposed angiotensin II-induced contraction and ERK1/2 activation, independent of superoxide production. These findings might be of importance in the treatment of vascular disorders.

Hypertension Research (2010) 33, 857-866; doi:10.1038/hr.2010.83; published online 10 June 2010

Keywords: angiotensin II; blood flow; endothelium; ERK1/2; vascular remodeling

\section{INTRODUCTION}

Angiotensin II is a vasoactive hormone-inducing vasoconstriction, ${ }^{1}$ cell growth ${ }^{2}$ and migration, as well as extracellular matrix deposition. ${ }^{3}$ Angiotensin II also has a central role in the functional and structural integrity of the vascular wall and in the pathogenesis of cardiovascular disease. ${ }^{4,5,6}$

Chronic changes in blood flow occur in physiological conditions such as exercise, pregnancy and postnatal development, as well as in pathological conditions such as arterial occlusive diseases, diabetes and hypertension. ${ }^{7,8}$ These changes in flow induce remodeling of the vascular wall to adapt arterial wall strain to the new hemodynamic conditions, as described in large ${ }^{9}$ and small (resistance) arteries. ${ }^{7,8}$

In large arteries, a chronic reduction in blood flow induces inward remodeling associated with intimal invasion. ${ }^{9}$ In small resistance arteries (RAs), a chronic reduction in blood flow induces inward remodeling without intimal invasion. Indeed, this remodeling is the consequence of the unbalanced arterial contractility resulting from reduced stimulation of the endothelium by flow-generated shear stress. This contraction is then stabilized by the activation of tissuetransglutaminases. ${ }^{10}$ Using mice lacking tissue angiotensin I-converting enzyme, Hilgers et al. ${ }^{11}$ showed that this enzyme has a major role in hyperplasic inward remodeling of the carotid artery after blood flow cessation. In mesenteric RAs, we have previously shown that acute stimulation of the endothelium by flow (shear stress) induces vasodilation, which is modulated by the local production of angiotensin II. ${ }^{12,13}$ Furthermore, we found that angiotensin II activation through type 2 receptor (AT2R) activation is directly involved in flowmediated dilation. ${ }^{12,14,15}$ We have also shown that angiotensin II induces hypertrophy through its type 1 receptor (AT1R) and extracellular signal-regulated kinase (ERK) $1 / 2$ activation, without being

${ }^{1}$ INSERM U771, Angers, France; ${ }^{2}$ CNRS UMR 6214, Angers, France; ${ }^{3}$ Université d'Angers, Angers, France; ${ }^{4} \mathrm{CHU}$ d'Angers, Angers, France and ${ }^{5}$ INSERM U892, CRCNA, Angers, France

Correspondence: Dr D Henrion, UMR CNRS 6214 - INSERM 771, Faculté de Médecine, Angers, 49045, France.

E-mail: Daniel.henrion@univ-angers.fr

Received 26 January 2010; revised 4 April 2010; accepted 7 April 2010; published online 10 June 2010 
involved in diameter enlargement, in RAs submitted chronically to increased blood flow. ${ }^{16}$ Thus, in response to a chronic rise in flow, diameter increases because of activation of the NO-cGMP pathway, ${ }^{17}$ and wall mass increases after activation of AT1R and ERK1/2. ${ }^{16}$ However, inward remodeling because of a chronic decrease in flow involves a different mechanism, defined primarily by over activity of the contractile agents because of flow (shear stress) reduction.

We hypothesized that angiotensin II has a role in inward remodeling because of blood flow reduction, as locally produced angiotensin II may remain unbalanced after blood flow reduction. We also tested the role of superoxide anions and ERK1/2 in inward remodeling, as these molecules are involved in angiotensin II-mediated contraction in mesenteric RAs ${ }^{18}$ as well as in cell migration. ${ }^{19}$ We used a model previously described in rats ${ }^{20,21}$ and mice, ${ }^{22,23}$ which allows comparison of RAs submitted to low blood flow to adjacent arteries with normal flow (NF) in the same physiological conditions in vivo, without changes in hemodynamic conditions or hormonal environment. It should be noted that, in this model, flow decreases without changes in blood pressure or hemodynamic environment. Thus, flow (shear stress) decreases without any change in arterial wall strain, as observed in hypertension, whereas in models of hypertension, both pressure and flow are affected. The involvement of flow, per se, in inward remodeling remains enigmatic. We tested the role of angiotensin II in this remodeling through the use of angiotensin I-converting enzyme inhibition with perindopril and AT1R blockade with candesartan. Hydralazine was used as a control nonspecific vasodilator treatment. ${ }^{24}$

\section{METHODS}

\section{Animals}

Twelve-week-old Wistar rats (Iffa-Credo, L'Arbresle, France) were anesthetized (isoflurane, 2.5\%) and pretreated with buprenorphine (Temgesic; $0.1 \mathrm{mg} \mathrm{kg}^{-1}$, subcutaneous). A loop of intestine was then exposed, and local mesenteric artery blood flow was surgically reduced, as previously described. ${ }^{17}$ Briefly, second-order arterial branches were ligated with 7-0 surgical silk threads to reduce flow in the first-order feeding artery (low flow (LF) artery). Control (NF) vessels were other first-order mesenteric arteries obtained from the same animal.

Rats were treated with perindopril $\left(2 \mathrm{mg} \mathrm{kg}^{-1}\right.$ per day; $n=12$ rats), candesartan $\left(2 \mathrm{mg} \mathrm{kg}^{-1}\right.$ per day; $n=12$ rats), hydralazine $\left(200 \mathrm{mgl}^{-1}\right.$ per day in drinking water; $n=12$ rats) or tap water (control group, $n=12$ ) for 1 week. Treatments were started 1 day before surgery.

In another series of experiments, rats were treated with the SOD-mimetic tempol (4-hydroxy-2,2,6,6-tetramethyl piperidinoxyl; $20 \mathrm{mg} \mathrm{kg}^{-1}$ per day; $n=8$ rats), with the $\mathrm{NAD}(\mathrm{P}) \mathrm{H}$-oxidase inhibitor apocynin $\left(50 \mathrm{mg} \mathrm{kg}^{-1}\right.$ per day in drinking water; $n=8$ rats) or with the ERK1/2 inhibitor U0126 (1,4-diamino2,3-dicyano-1,4-bis (2-aminophenylthio) butadiene; $30 \mathrm{mg} \mathrm{kg}^{-1}$ per day, subcutaneous; $n=8$ rats).

In another group of experiments, mice lacking the gene coding for eNOS (eNOS $-/-)$ and their controls $(+/+)(n=6$ per group) were subjected to the protocol described above.

After 7 days, rats were re-anesthetized, and arterial blood pressure was measured in the left femoral artery. ${ }^{25}$ The animals were then killed by $\mathrm{CO}_{2}$ inhalation. The gut was excised, and the mesenteric arteries were dissected as previously described. ${ }^{26}$ Each arterial segment was divided into two parts, proximal for the functional study and distal for histological and biochemical analyses.

The procedure followed for the care and the euthanasia of the study animals was in accordance with the European Community Standards on the Care and Use of Laboratory Animals (Ministère de l'Agriculture, France, authorization no. 6422), and the protocol was approved by the Regional (Pays de la Loire) Ethical Committee.

\section{Isolated mesenteric artery}

Segments of mesenteric arteries ( $2 \mathrm{~mm}$ long) were dissected and mounted on a wire-myograph (DMT, Aarhus, Denmark) as previously described. ${ }^{27-29}$ Briefly, two tungsten wires $(25 \mu \mathrm{m}$ diameter) were inserted into the lumens of the arteries and fixed to a force transducer and a micrometer. Arteries were bathed in a physiological salt solution maintained at $37^{\circ} \mathrm{C}, \mathrm{pH} 7.4\left(\mathrm{pO}_{2} 160 \mathrm{~mm} \mathrm{Hg}\right.$, $\mathrm{pCO}_{2} 37 \mathrm{~mm} \mathrm{Hg}$ ). ${ }^{30}$ Wall tension was applied as previously described. ${ }^{31} \mathrm{In}$ brief, arteries were set to the baseline circumference $\mathrm{L}_{0}$, where $\mathrm{L}_{0}=0.9 \mathrm{~L}_{100}$ is the internal circumference that the artery would have in vivo when relaxed and under a transmural pressure of $100 \mathrm{~mm} \mathrm{Hg}$. The near-maximal active wall tension of the vessel is developed at this circumference. Vessels were then allowed to stabilize for $1 \mathrm{~h}$. Artery viability was tested using a potassium-rich solution $\left(80 \mathrm{mmoll}^{-1}, 80 \mathrm{~K}\right.$ physiological salt solution). Angiotensin II $\left(10 \mathrm{nmoll}^{-1}\right)$, the thromboxane $\mathrm{A}_{2}$ stable mimetic U46619 $\left(1 \mu \mathrm{moll}^{-1}\right)$ or phenylephrine (PE; $1 \mu \mathrm{moll}^{-1}$ ) was then added to the bath to measure arterial contractility. Acetylcholine (Ach; $1 \mathrm{nmoll}^{-1}-10 \mu \mathrm{moll}^{-1}$ )-induced relaxation was then obtained after PE-induced preconstriction (50\% of maximal contraction).

In a separate series of experiments, arteries were pretreated with the ERK1/2 blocker PD98059 $\left(10 \mu \mathrm{moll}^{-1}\right)$ before inducing contraction with angiotensin II $\left(10 \mathrm{nmoll}^{-1}\right)$ or PE $\left(1 \mu \mathrm{moll}^{-1}\right)$.

\section{Histology}

A segment of each mesenteric artery was dissected, cannulated at one end and ligated at the other end. The artery was then bathed in $\mathrm{Ca}^{2+}$-free physiological salt solution containing $10^{-5} \mathrm{moll}^{-1}$ sodium nitroprusside. Pressure was set at $75 \mathrm{~mm} \mathrm{Hg}$, and the artery was fixed in a $10 \%$ buffered formaldehyde solution as previously described. ${ }^{32}$ Cross-sections $(10 \mu \mathrm{m})$ were stained with orcein. The external diameter, lumen diameter and media thickness were determined after image acquisition (Olympus T100 microscope Olympus France, Rungis, France, Sony camera, Sony, Rungis, France) and analyzed using the Histolab software (Microvision, Paris, France) for calculations of cross-sectional area, as previously described. ${ }^{33}$

\section{Western blot analysis of eNOS, AT2R, AT1R, p38, p42 and p44}

Arterial tissues from two rats were pooled (two first-order arteries for NF and four for LF) and then homogenized, as previously described. ${ }^{17}$ Proteins ( $20 \mu \mathrm{g}$ of total protein from each sample) were separated by sodium dodecyl sulfatepolyacrylamide gel electrophoresis (Mini gel protean II system (Bio-Rad, Marnes la Coquette, France), $100 \mathrm{~V}$, with $300 \mathrm{ml}$ of $25 \mathrm{mmoll}^{-1}$ Tris, $192 \mathrm{mmoll}^{-1}$ glycine and $0.1 \%$ sodium dodecyl sulfate), using a $4 \%$ stacking gel followed by an $8 \%$ running gel. After migration, proteins were transferred ( $40 \mathrm{~V}$, overnight at $4{ }^{\circ} \mathrm{C}$, with $800 \mathrm{ml}$ of $25 \mathrm{mmoll}^{-1}$ Tris, $192 \mathrm{mmoll}^{-1}$ glycine, $0.01 \%$ sodium dodecyl sulfate and $20 \%$ methanol) to PVDF blotting membranes (Amersham, Saclay, France). Membranes were then washed in TBS-T buffer (composed of $10 \mathrm{mmoll}^{-1}$ Tris/base $\mathrm{pH} 7.5,0.1 \mathrm{moll}^{-1} \mathrm{NaCl}, 1 \mathrm{mmoll} \mathrm{l}^{-1}$ EDTA and $0.1 \%$ Tween 20$)$ and blocked for $2 \mathrm{~h}$ at room temperature $(10 \%$ bovine serum albumin in TBS-T). Membranes were incubated for $90 \mathrm{~min}$ at room temperature with the primary antibody (Transduction Laboratories, Franklin Lakes, NJ, USA: eNOS, p38, phospho-p38, p42, p44, phospho p42/ p44 and Santa Cruz Biotechnology, Santa Cruz, CA, USA: AT2R and AT1R), washed again (three times for $10 \mathrm{~min}$ ), and incubated with either anti-mouse or anti-rabbit peroxidase-conjugated antibody (Amersham) in TBS-T. Membranes were washed (three times for $10 \mathrm{~min}$ ), and labeling was visualized using the ECL-Plus Chemiluminescence kit (Amersham). ${ }^{34}$

\section{Detection of reactive oxygen species (ROS) using confocal microscopy in RAs}

Superoxide detection was performed on transverse cross-sections ( $7 \mu \mathrm{m}$ thick) incubated with dihydroethydine as previously described. ${ }^{35,36}$ Positive staining was visualized using confocal microscopy and QED-image software (Solamere Technology, Salt Lake City, UT, USA). Image analysis was performed using Histolab (Microvision). Pixel quantification was executed as previously described. ${ }^{14}$ Sections of mesenteric artery treated for $2 \mathrm{~h}$ with lipopolysaccharide in vitro at $37^{\circ} \mathrm{C}$ in a physiological salt solution served as a positive control. 
A negative control was obtained by omitting dihydroethydine or by adding tempol to the section $15 \mathrm{~min}$ before dihydroethydine.

\section{Statistical analysis}

Results are expressed as means \pm s.e.m. Significance of the difference between arteries was determined by analysis of variance (one-factor analysis of variance or analysis of variance for consecutive measurements, when appropriate). Means were compared by paired $t$-test or by the Bonferroni test for multigroup comparisons. Values of $P<0.05$ were considered to be significant.

\section{Drugs}

Candesartan was kindly provided by Astra-Zeneca (Göteborg, Sweden) and Perindopril by Servier (Paris, France). U0126 was purchased from LC Labora- tories (Woburn, MA, USA). Other compounds were purchased from Sigma-Aldrich (Lyon, France).

\section{RESULTS}

\section{Animals}

Mean arterial blood pressure $(101 \pm 4 \mathrm{~mm} \mathrm{Hg}$ in control rats, $n=12)$ was not significantly affected by hydralazine $(99 \pm 5 \mathrm{~mm} \mathrm{Hg}, n=12)$, candesartan $(103 \pm 5 \mathrm{~mm} \mathrm{Hg}, n=12)$, perindopril $(94 \pm 6 \mathrm{~mm} \mathrm{Hg}$, $n=12)$, tempol $(103 \pm 4, n=8)$, apocynin $(105 \pm 6 \mathrm{~mm} \mathrm{Hg}, n=8)$ or $\mathrm{U} 0126$ (96 $\pm 7 \mathrm{~mm} \mathrm{Hg}, n=8)$.

\section{Arterial diameter and cross-sectional area}

In NF arteries, internal diameter was not significantly modified by perindopril, candesartan or hydralazine (Figure 1a). Arterial

a

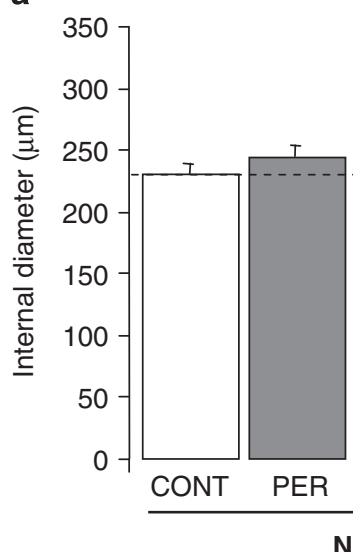

Internal arterial diameter
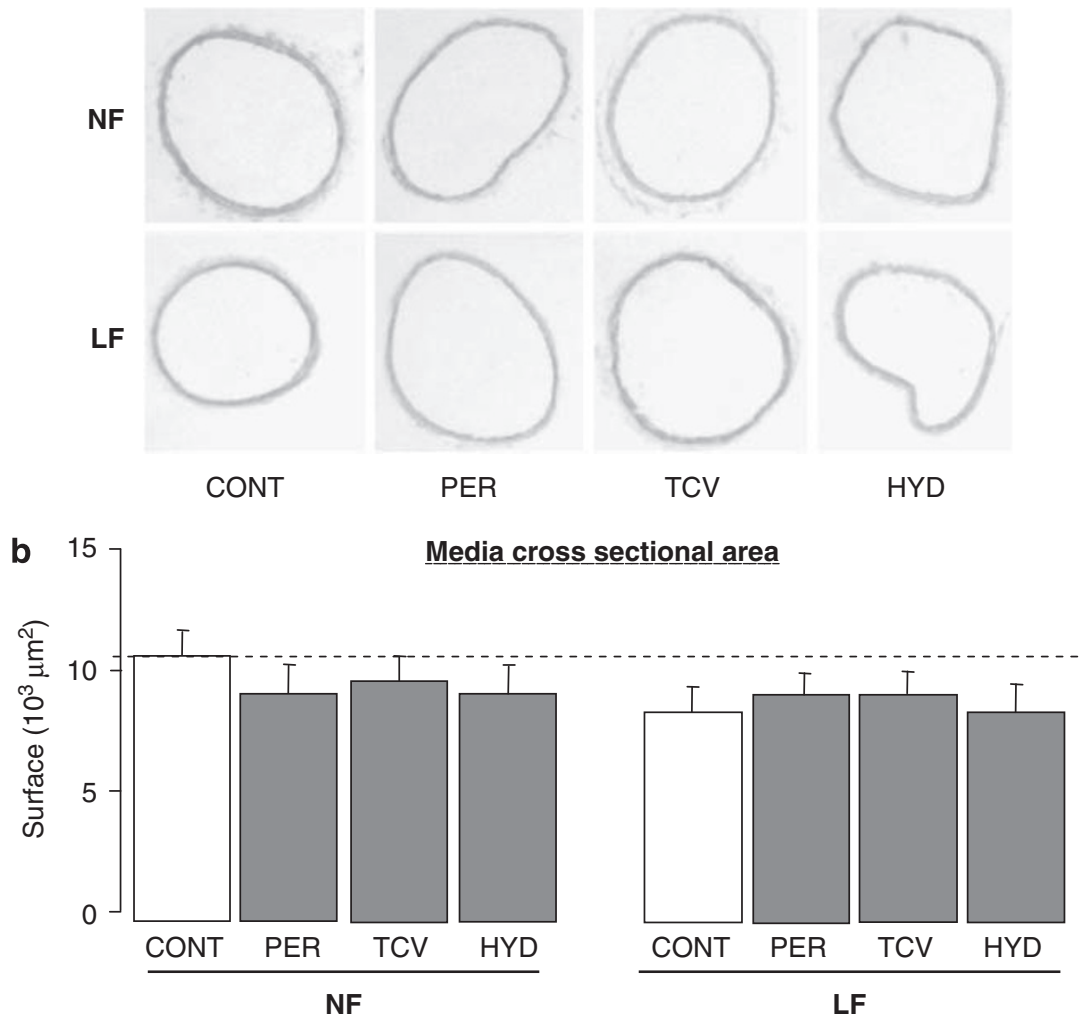

Figure 1 Internal arterial diameter (a) and medial cross-sectional area (b) of mesenteric RAs exposed chronically to a LF blood in vivo. LF arteries were compared with NF arteries. Arteries were isolated from rats treated with perindopril (PER), candesartan (TCV), hydralazine (HYD) or water (CONT: control). Means \pm s.e.m. are presented ( $n=12$ rats per group). ${ }^{*} P<0.05$, LF vs. NF. ${ }^{*} P<0.05$, TCV, PER or HYD compared with CONT. 
diameter was significantly lower in LF as compared with NF arteries in control and hydralazine-treated animals. In perindopril- and candesartan-treated rats, LF artery diameter was not significantly different from control NF arteries, suggesting that LF remodeling was prevented by the treatments (Figure 1b).

Cross-sectional area (Figure 1b) was not significantly modified by perindopril, candesartan or hydralazine in NF and LF arteries.

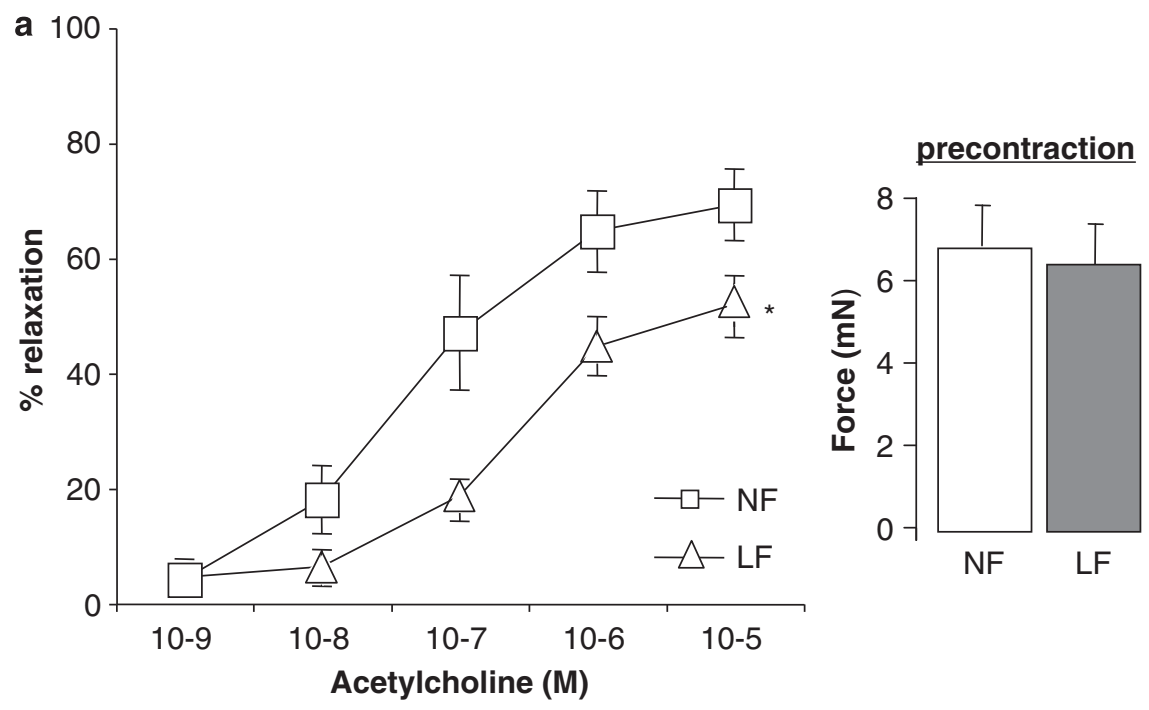

b

IC50 ( $\mu \mathrm{mol} / \mathrm{L})$
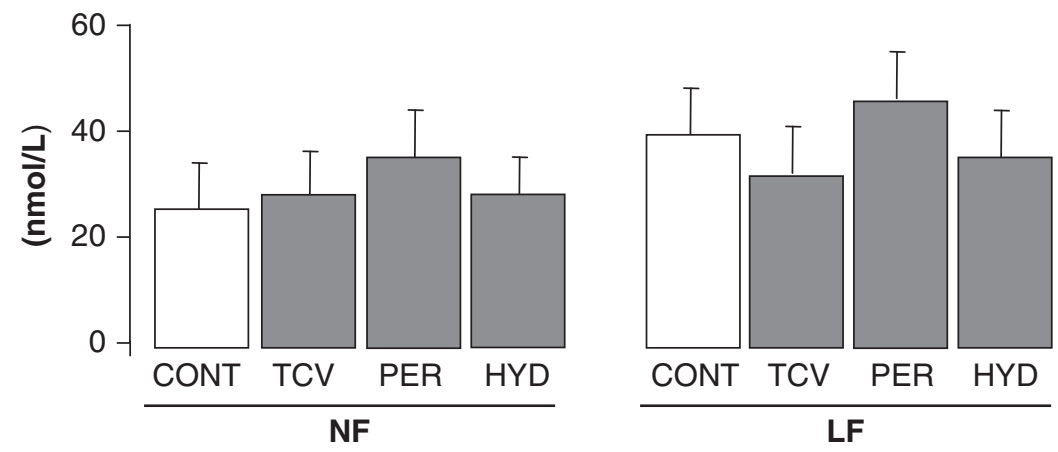

c

I-max
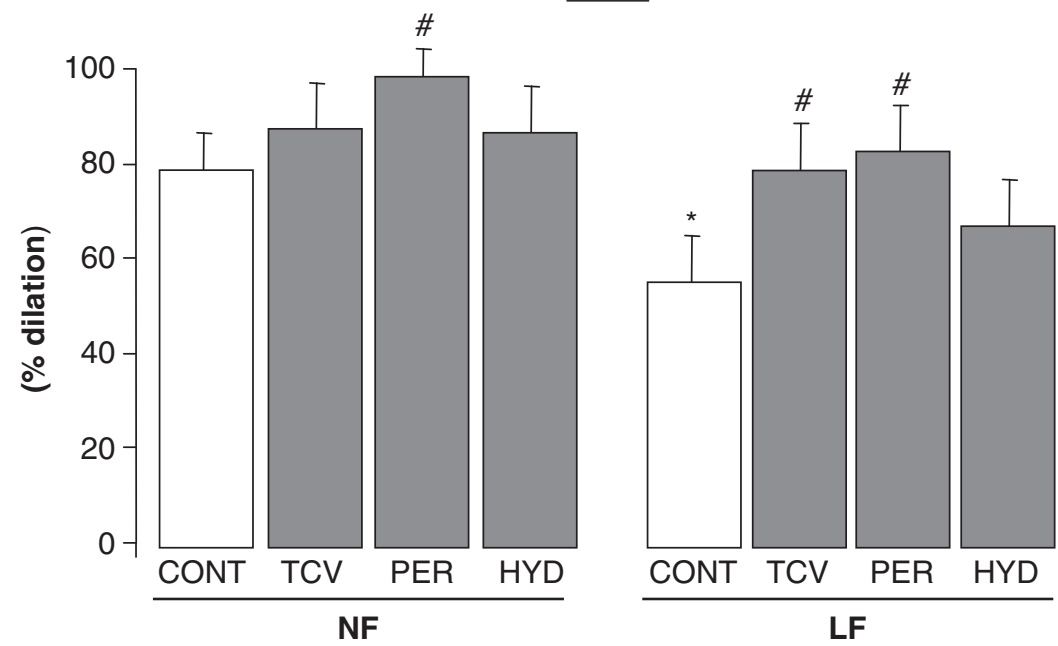

Figure 2 Ach-induced relaxation (a) obtained in mesenteric RAs submitted to a chronic decrease in blood flow (LF) compared with control arteries exposed to NF. Relaxation is expressed as percentage of relaxation of PE-induced precontraction (shown in the right insert). Rats were treated with perindopril (PER), candesartan (TCV), hydralazine (HYD) or water (CONT: control). IC50 (b) and Imax (c) were calculated for each individual concentration response curve. Means \pm s.e.m. are presented ( $n=12$ rats per group). ${ }^{*} P<0.05$, LF vs. NF ${ }^{\#} P<0.05$, TCV, PER or HYD compared with CONT. 
Endothelium-dependent relaxation and eNOS expression level Ach induced a significant concentration-dependent relaxation of NF and LF arteries (Figure 2a). Ach-induced relaxation in NF was not affected by hydralazine or candesartan and was enhanced by perindopril. Indeed, Imax, but not $\mathrm{IC}_{50}$, was increased by perindopril (Figures $2 \mathrm{~b}$ and $\mathrm{c}$ ). In LF arteries, Ach-induced relaxation was significantly lower than in NF arteries in control arteries (the Imax decreased but the IC50 did not, Figures $2 \mathrm{~b}$ and c). In rats treated with perindopril or candesartan, Ach-induced relaxation was equivalent in NF and LF arteries and significantly higher than in control NF arteries.

In NF arteries, the eNOS expression level was not significantly affected by perindopril, candesartan or hydralazine. The expression level of eNOS was lower in LF arteries than in NF vessels (Figure 3a). In LF arteries from perindopril- or candesartan-treated rats, the eNOS expression level was not significantly different from that in NF arteries. However, eNOS expression was significantly higher than eNOS expression in LF vessels.

To assess the role of a drop in eNOS expression on the LF-induced reduction in diameter, we submitted the mesenteric RAs of eNOS KO mice to ligation. The absence of eNOS did not affect the decrease in diameter or the cross-sectional area in LF arteries (Figures $3 \mathrm{~b}$ and c).

\section{Role of ROS in remodeling}

Superoxide production was significantly higher in LF as compared with NF arteries in control rats (Figure 4a). Hydralazine, perindopril

a eNOS expression level
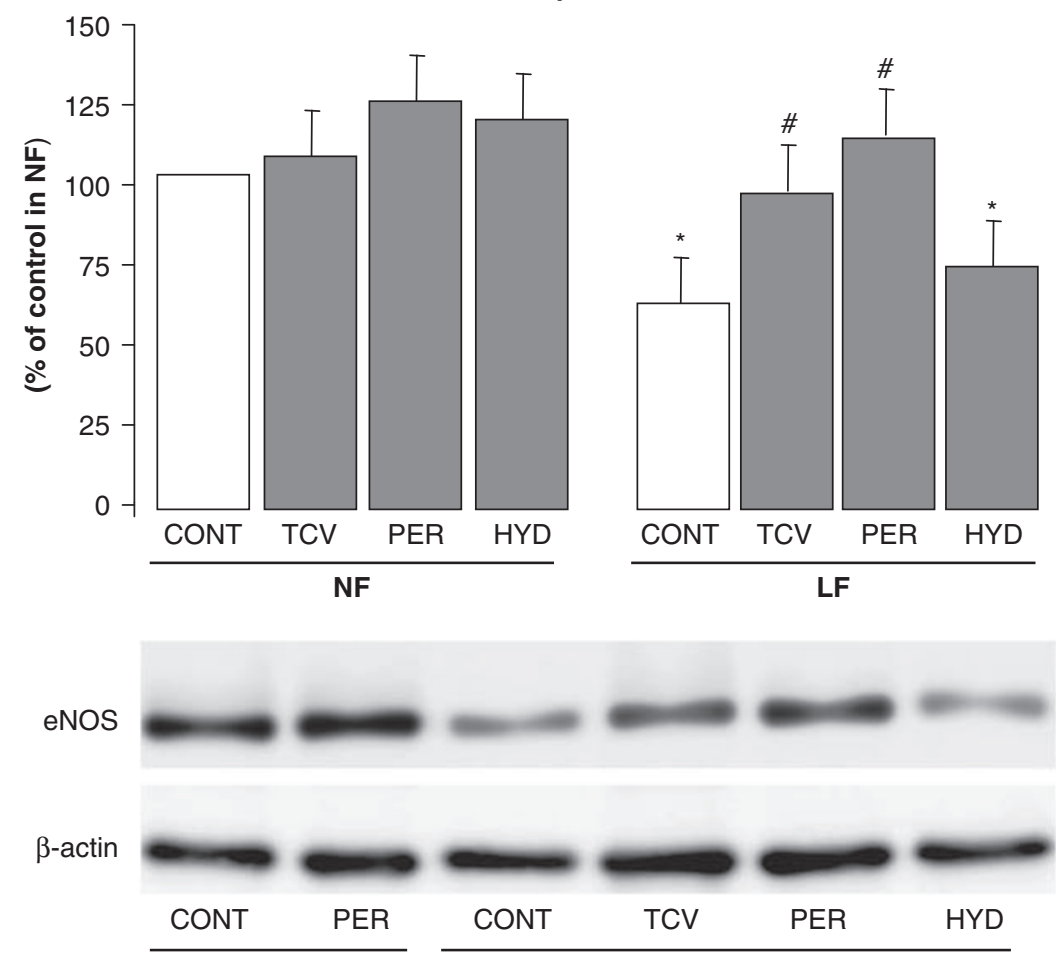

NF

LF
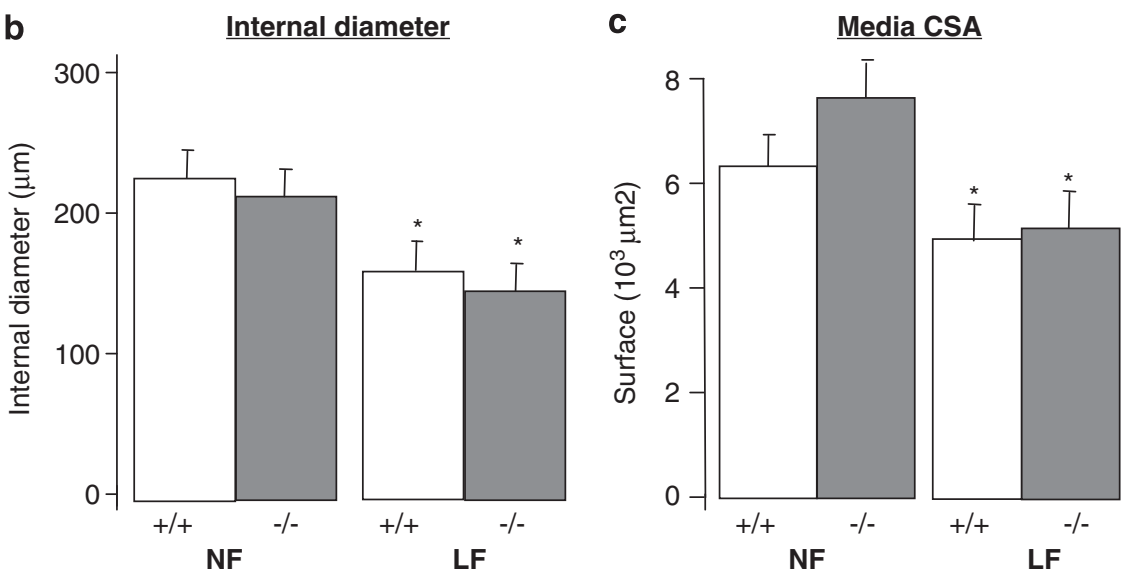

Figure 3 eNOS expression level (a) obtained in mesenteric RAs exposed to a chronic decrease in blood flow (LF), compared with control arteries exposed to NF. Rats were treated with perindopril (PER), candesartan (TCV), hydralazine (HYD) or water (CONT: control). Means \pm s.e.m. are presented ( $n=12$ rats per group). (b, c) show internal arterial diameter and cross-sectional area as measured in LF and NF arteries isolated from mice lacking eNOS ( $-I-)$ and in controls $(+/+)$. Means \pm s.e.m. are presented ( $n=6$ mice per group). ${ }^{*} P<0.05$, LF vs. NF ${ }^{\#} P<0.05$, TCV, PER or HYD compared with CONT (a). 


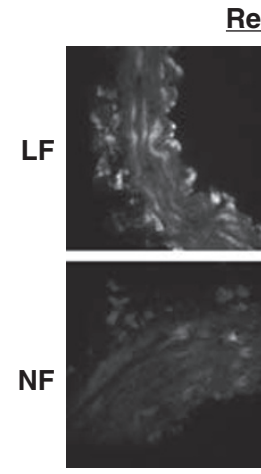

CONT
Reactive oxygen species (DHE staining)

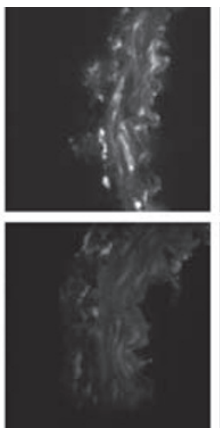

PER

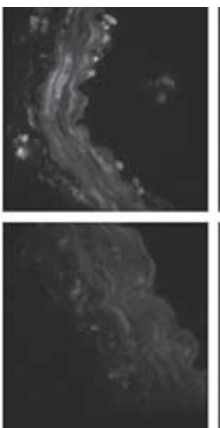

TCV
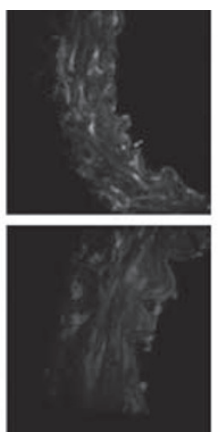

HYD
Pos. control
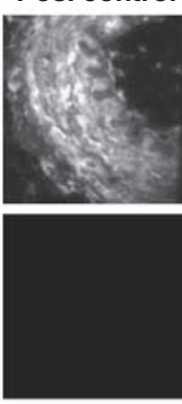

Neg. control

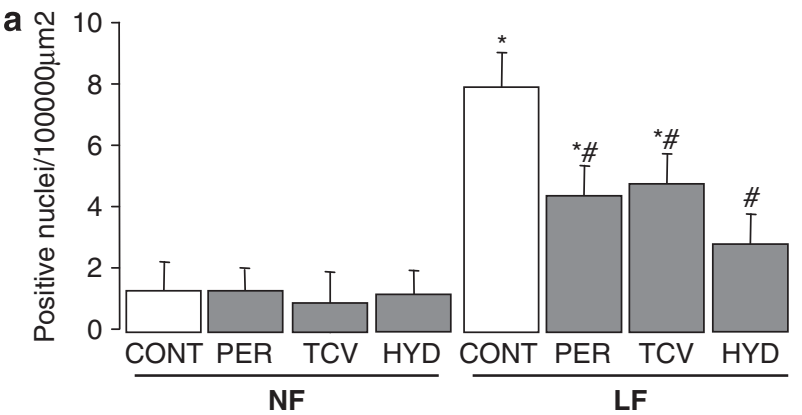

b

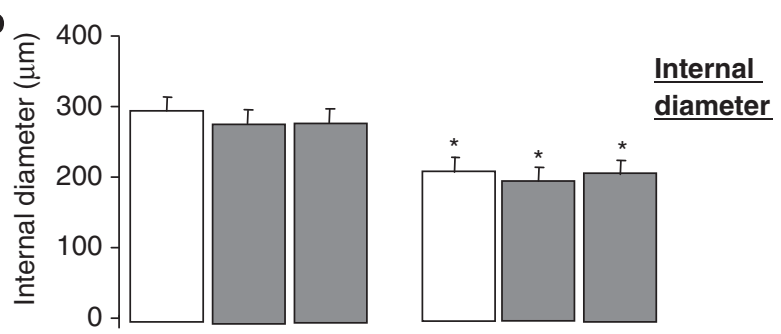

c

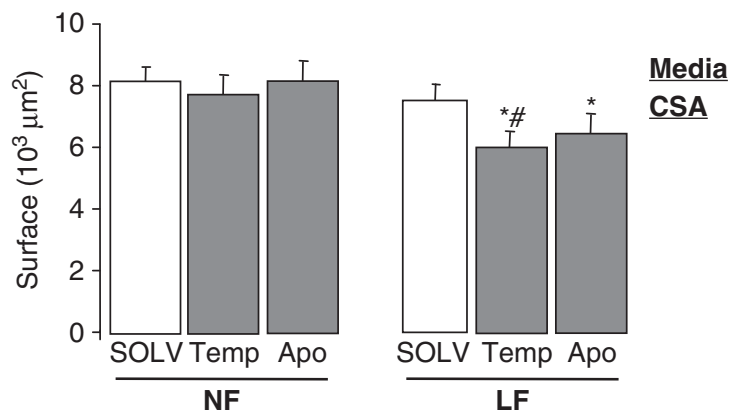

Figure 4 ROS visualized using dihydroethydine microuorography (a) in mesenteric RAs exposed to a chronic decrease in blood flow (LF), compared with NF arteries. ROS level was quantified by image analysis in rats treated with perindopril (PER), candesartan (TCV), hydralazine (HYD) or water (CONT: control). Means \pm s.e.m. are presented ( $n=12$ rats per group). (b, c) show internal arterial diameter and cross-sectional area measured in LF and NF mesenteric arteries obtained from rats treated chronically with tempol (Temp), apocynin (Apo) or water (SOLV). Means \pm s.e.m. are presented ( $n=8$ per group). ${ }^{*} P<0.05$, LF vs. NF ${ }^{\#} P<0.05$, TCV, PER or HYD compared with CONT (a).

and candesartan significantly reduced superoxide production in LF arteries, although in LF arteries from perindopril- and candesartantreated rats, superoxide levels remained significantly higher than in NF arteries (Figure 4a).

To test the role of superoxide in the diameter reduction of LF arteries, rats were treated chronically with the SOD mimetic tempol or with the $\mathrm{NAD}(\mathrm{P}) \mathrm{H}$-oxidase inhibitor apocynin. These treatments did not prevent the decrease in diameter, but they did induce a reduction in media cross-section in LF arteries (Figures $4 \mathrm{~b}$ and c).

\section{Vasoconstrictor tone}

As the LF-associated reduction in diameter was attributed to unopposed vasoconstrictor tone, we measured contraction induced by $\mathrm{KCl}(80 \mathrm{~mm}), \mathrm{U} 46619\left(1 \mu \mathrm{moll}^{-1}\right), \mathrm{PE}\left(1 \mu \mathrm{moll}^{-1}\right.$, Figure $\left.5 \mathrm{a}\right)$ and angiotensin II $\left(10 \mathrm{nmoll}^{-1}\right.$, Figure $\left.5 \mathrm{~b}\right)$ in LF vessels. In isolated NF and LF arteries, $\mathrm{KCl}, \mathrm{U} 46619 \mathrm{PE}$ and angiotensin II induced a significant contraction.

In NF arteries, the contraction induced by PE was not modified by perindopril, candesartan or hydralazine (Figure 5a). Similarly, 
a
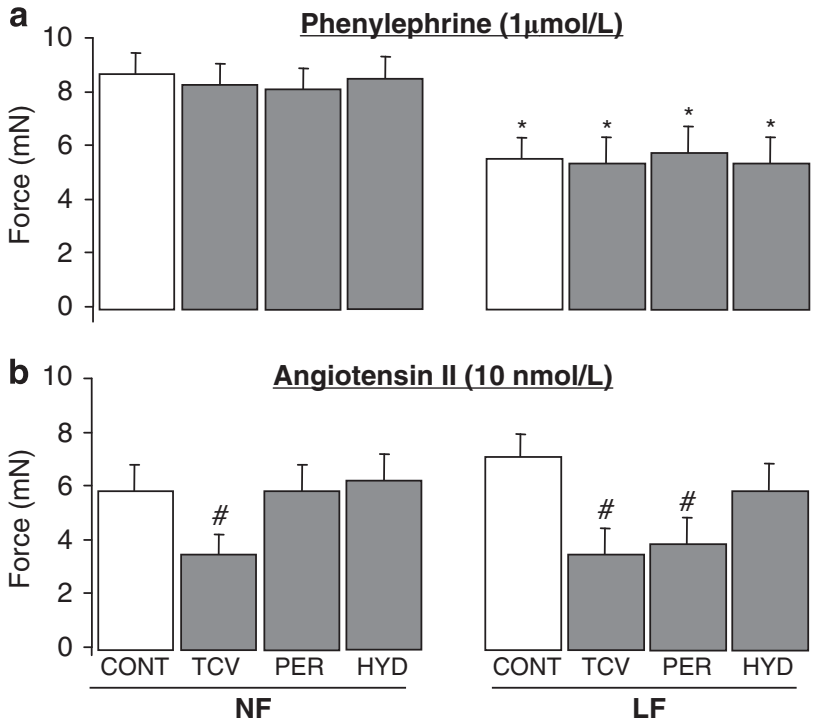

c

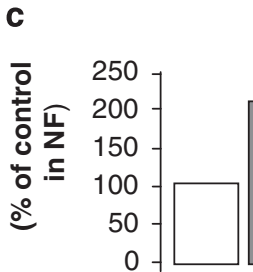

AT1R expression level
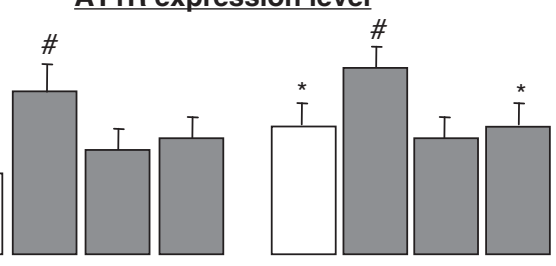

d
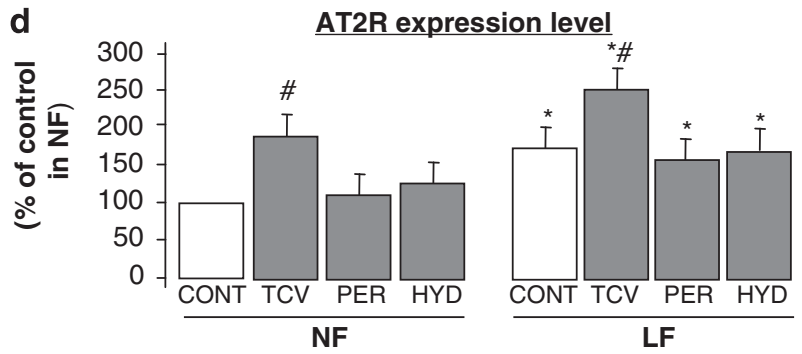

Figure 5 Contraction induced by PE (a) and angiotensin II (b) in mesenteric RAs exposed to a chronic decrease in blood flow (LF), as compared with NF arteries. AT1R (c) and AT2R (d) expression levels were measured using western blot. Rats were treated with perindopril (PER), candesartan (TCV), hydralazine (HYD) or water (CONT: control). Means \pm s.e.m. are presented ( $n=12$ rats per group). ${ }^{*} P<0.05$, LF vs. NF. ${ }^{\#} P<0.05$, TCV, PER or HYD compared with CONT.

contraction induced by $\mathrm{KCl}$ and $\mathrm{U} 46619$ was not affected by any of the drugs (data not shown). In the same arteries, the contraction induced by angiotensin II was not modified by chronic treatment with perindopril or hydralazine, but it was reduced by chronic candesartan treatment (Figure 5b).

In LF arteries, the contraction induced by PE but not that induced by angiotensin II was significantly lower than in NF vessels (Figures 5a and $\mathrm{b})$. The contraction induced by $\mathrm{KCl}(8.2$ vs $5.1 \mathrm{mN}, P<0.01)$ and by $\mathrm{U} 46619(9.2$ vs $5.6 \mathrm{mN}, P<0.01)$ was also weaker in $\mathrm{LF}$ as compared with NF arteries. Perindopril, candesartan and hydralazine did not affect PE- (Figure 5a), KCl- (data not shown) or U46619-induced contraction (data not shown) in LF arteries. Angiotensin II-mediated contraction was significantly reduced in LF arteries from perindopriland candesartan-treated rats, as compared with controls (Figure 5b, right panel). Hydralazine did not affect angiotensin II-mediated contraction in LF arteries.
The expression of angiotensin II receptors AT1R (Figure 5c) and AT2R (Figure 5d) was higher in LF than in NF arteries. Perindopril and hydralazine did not significantly affect the level of expression in NF and LF arteries. In contrast, candesartan increased AT1R and AT2R levels in both vessels.

As we have previously shown ${ }^{16}$ that angiotensin II-induced contraction was mediated by the activation of ERK1/2 in mesenteric RAs, we tested the effect of ERK1/2 blockade on vascular contractility in LF arteries. Indeed, the inhibition of ERK1/2 by PD98059 decreased angiotensin II-induced contraction in NF arteries and suppressed the phenomenon in LF vessels (Figure 6a).

\section{Mitogen-activated protein kinase activation}

The ratio of phospho-ERK1/2 to ERK1/2 (p42 and p44) was significantly higher in LF arteries compared with NF arteries (Figure 6a). Similarly, the phospho-p38/p38 ratio was also higher in LF than in NF arteries in all study groups (Figure $6 \mathrm{~b}$ ).

In LF arteries, ERK1/2 activation (phospho-ERK1/2 to ERK1/2 ratio) was lower in rats treated with perindopril or candesartan as compared with control rats (Figure 6a). Perindopril, candesartan and hydralazine did not affect the phospho-p38/p38 ratio in LF arteries (Figure 6c).

As perindopril and candesartan reduced ERK1/2 activation through a chronic decrease in flow while ERK1/2 inhibition preferentially reduced angiotensin II-induced contraction, we tested the effect of a chronic blockade of ERK1/2 on LF-mediated remodeling. The ERK1/2 inhibitor U0126 was administered to rats submitted to mesenteric artery ligation. This treatment prevented an LF-related reduction in diameter. Indeed, in U0126-treated rats arterial diameter was similar in LF and NF arteries (Figure 7a), suggesting that the treatment prevented changes in diameter. Cross-section was not affected in NF vessels, whereas this parameter was reduced in LF arteries from U0126-treated rats (Figure $7 \mathrm{~b}$ ).

\section{DISCUSSION}

The model used allows the study of comparable RAs submitted to low or normal blood flow in vivo without changes in physiological hemodynamic conditions, in rats and mice. ${ }^{17,20,22,23} \mathrm{~A}$ reduction in blood flow induces a reduction in diameter, with or without arterial wall hypotrophy, decreased endothelium (NO)-dependent dilation and reduced contractility. ${ }^{20,22,23,32,33,37}$ In addition to being a model that mimics the remodeling that occurs in hypoperfused tissues, LF remodeling has been shown to be equivalent to the eutrophic inward remodeling observed in several types of hypertension, such as endothelin-1-dependent hypertension and L-NAME-induced hypertension. ${ }^{10}$

The main finding of this study is that the diameter reduction because of a decrease in blood flow in RAs was prevented by perindopril and candesartan, but not by hydralazine, suggesting selective involvement of angiotensin II and of its AT1R. The AT2R was not involved in this remodeling (see Supplementary information).

This remodeling was associated with reduced Ach-dependent dilation and decreased eNOS protein expression. Similarly, in LF arteries bradykinin type 2 receptor expression was decreased, whereas bradykinin type 1 receptor was unaffected (see Supplementary information). Both perindopril and candesartan but not hydralazine prevented the decrease in endothelium-dependent dilation and in eNOS expression level in LF arteries. Nevertheless, these effects cannot explain why both drugs prevented the decrease in diameter because LF artery diameter reduction was not prevented in mice lacking the gene coding for eNOS. This finding is in agreement with our previous study. ${ }^{17}$ 


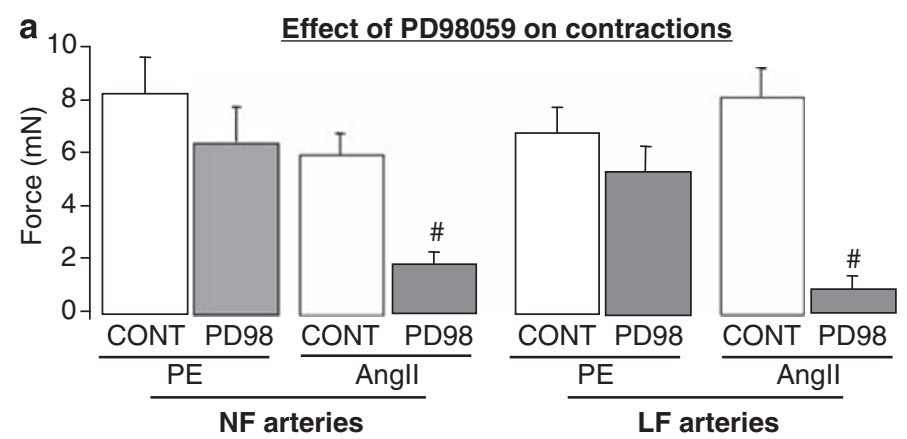

b

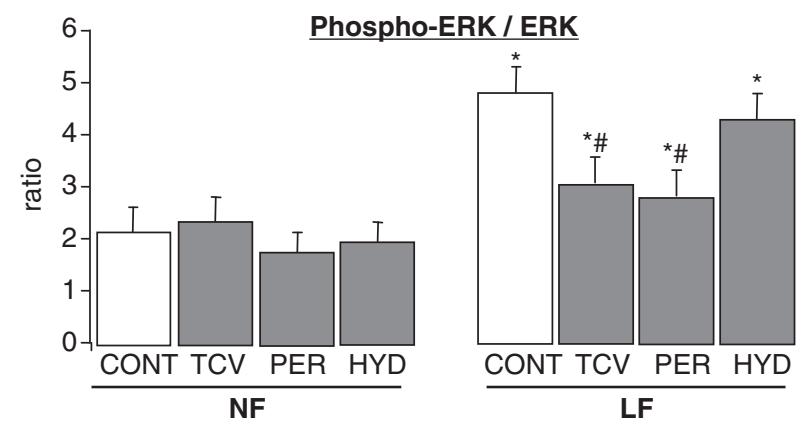

c
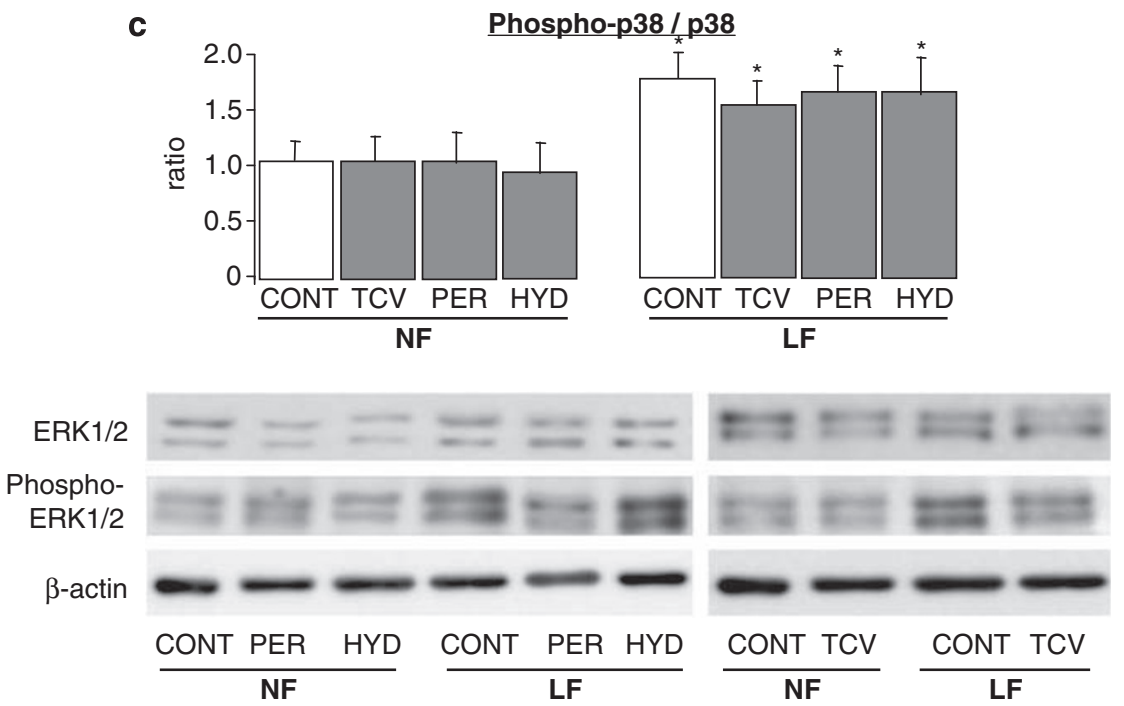

Figure 6 Effect of blocking ERK1/2 with PD98059 $\left(10 \mu \mathrm{moll}^{-1}\right)$ on PE- and angiotensin II-induced contraction (a). Activation (ratio of the phosphorylated form per total) of mitogen-activated protein (MAP) kinases ERK1/2 (p42 and p44) and p38 (b) in mesenteric RAs exposed to a chronic decrease in blood flow (LF), as compared with NF arteries. Rats were treated with perindopril (PER), candesartan (TCV), hydralazine (HYD) or water (CONT: control). The lower panel shows typical blots of ERK1/2 (p44) and -actin. Means \pm s.e.m. are presented ( $n=12$ rats per group). ${ }^{*} P<0.05, \mathrm{LF} v s$. NF ${ }^{\#} P<0.05, \mathrm{TCV}, \mathrm{PER}$ or HYD, compared with CONT.

Furthermore, the absence of eNOS did not affect wall mass. In a previous study, ${ }^{17}$ we showed that chronic treatment of the rats with the NO synthesis blocker L-NAME did not prevent the diameter reduction in LF arteries and did not affect the medial surface. Nevertheless, both perindopril and candesartan improved endotheliumdependent dilation in LF arteries, in addition to preventing the reduction in diameter. This is in agreement with previous reports showing the positive effect of blocking the converting enzyme or the AT1R on endothelial function. ${ }^{38}$

ROS have a key role in the signaling pathways activated by angiotensin II in vascular cells. Therefore, we tested the involvement of ROS in LF remodeling. Indeed, we found that ROS production was increased in LF arteries compared with NF vessels. In rats chronically treated with perindopril, candesartan and hydralazine, ROS levels were reduced in LF arteries. Although hydralazine was the most efficient in reducing ROS levels in LF arteries, it did not affect the reduction in diameter, in contrast with perindopril and candesartan, suggesting that ROS might not be involved in this remodeling. This was confirmed in rats chronically treated with two different antioxidants, tempol and apocynin. Neither tempol nor apocynin affected the diameter reduction in LF arteries, confirming that ROS have no major effect in the process. In contrast, a significant reduction in media cross-sectional area was observed in LF arteries from rats treated with tempol or apocynin. This is in agreement with previous 

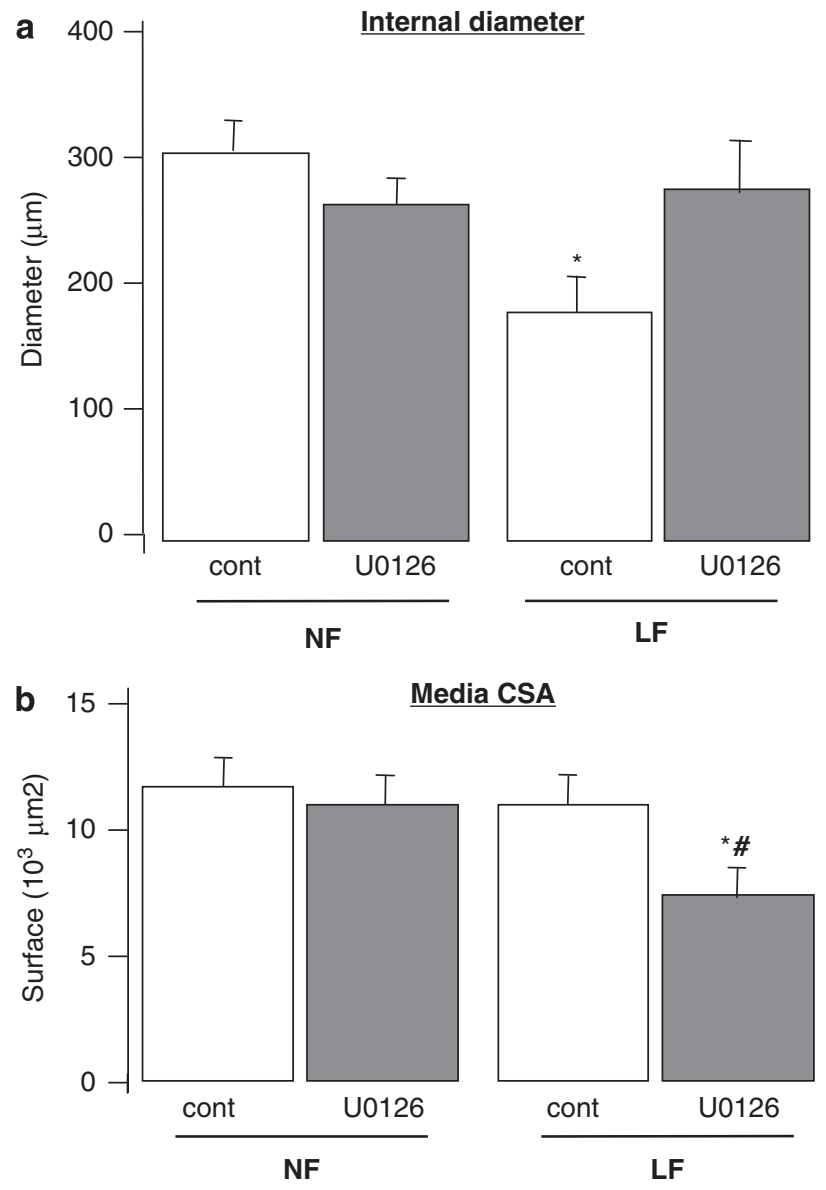

Figure 7 Effect of the ERK1/2 blocker U0126 (30 $\mathrm{mg} \mathrm{kg}^{-1}$ per day) on diameter changes (a) and medial cross-sectional area (CSA, $\mathbf{b})$ in NF and LF arteries. Means \pm s.e.m. are presented ( $n=8$ rats per group). ${ }^{*} P<0.05$, LF vs. NF ${ }^{\#} P<0.05$, U0126 vs CONT.

studies showing that the growth and proliferative activity induced by angiotensin II involve ROS generation. ${ }^{39}$ Finally, the rise in superoxide production in LF arteries might result from the initial macrophage infiltration after the decrease in blood flow. ${ }^{40}$

As previously shown, ${ }^{41}$ inward remodeling because of LF depends on the unopposed constrictor tone after the reduction in flow. Vasoconstrictor tone in RAs relies in large part on locally produced angiotensin II. ${ }^{12,15,42,43}$ In LF arteries, angiotensin II-induced contraction was strongly reduced in rats treated with perindopril or candesartan, but not in rats treated with hydralazine. In addition, both AT1R and AT2R expression levels were higher in LF as compared with NF arteries. Nevertheless, we may exclude a role of the AT2R in LF remodeling because chronic treatment with the AT1R blocker PD123319 or the absence of AT2R in mice did not prevent the reduction in diameter (see Supplementary information).

We have previously shown that angiotensin II-mediated contraction through the AT1R in mesenteric RAs requires ERK1/2 activation, ${ }^{13}$ and we found in this study that acute ERK1/2 blockade with PD98059 strongly reduced angiotensin II-induced contraction in LF arteries. In addition, we found that ERK1/2 phosphorylation was higher in LF than in NF arteries. Mitogen-activated protein kinase activity was not affected by perindopril, candesartan or hydralazine in NF arteries. However, in LF vessels, mitogen-activated protein kinase activity was decreased by perindopril and candesartan only. In addition, p38 phosphorylation was activated but not affected by the treatments.

Thus, unbalanced local vasoactive systems, mainly angiotensin II, ${ }^{42}$ increase the activation of ERK1/2 and p 38 in LF arteries. Nevertheless, angiotensin II and the activation of ERK1/2 seem to have the central role in the diameter reduction induced by the chronic decrease in blood flow.

Interestingly, in a previous work, we showed that in response to a chronic increase in flow, angiotensin II induces hypertrophy (through AT1R) without eliciting any change in diameter, ${ }^{16}$ whereas this study shows that in response to a decreased flow, angiotensin II controls the diameter change without obvious effects on wall mass. Thus, in ischemia (with reduced flow in ischemic tissues and a rise in flow in the surrounding tissues), blocking the AT1R might have a doubly beneficial effect, preventing any reduction in diameter in the ischemic zone and reducing hypertrophy in the feeding arteries that are sometimes recruited to vascularize the ischemic tissue.

The findings of this study are important with regard to pathological situations involving RA remodeling. In most cardiovascular and metabolic diseases, arterial remodeling occurs in association with changes in pressure and/or local blood flow. This is, for example, the case in hypertension, ${ }^{44}$ diabetes ${ }^{45}$ and in ischemic diseases. ${ }^{46}$ This study shows that the reduction in flow (shear stress), independent of pressure, induces inward remodeling through activation of angiotensin II and ERK1/2.

Treatment of cardiovascular diseases often involves ACEI or AT1Rblockers. Their effect on flow-mediated remodeling of RAs might present an advantage over other drug classes.

In conclusion, these findings show that in RAs, the diameter reduction because of chronic blood flow reduction involves local expression of angiotensin II. These findings provide further evidence that ACEI or AT1R-blockers might be helpful in the treatment of ischemic diseases.

\section{ACKNOWLEDGEMENTS}

Odile Dumont was supported in part by the Foundation for Medical Research (FRM: Fondation pour la Recherche Médicale), Paris, France. We thank the local Animal Care Unit of the University of Angers and Jérôme Roux, Pierre Legras and Dominique Gilbert for their kind help in treating the rats.

1 Henrion D, Laher I, Laporte R, Bevan JA. Further evidence from an elastic artery that angiotensin II amplifies noradrenaline-induced contraction through activation of protein kinase C. Eur J Pharmacol 1992; 224: 13-20.

2 Ruiz E, Redondo S, Padilla E, Gordillo-Moscoso A, Salaices M, Balfagon G, Tejerina T. Importance of intracellular angiotensin II in vascular smooth muscle cell apoptosis: inhibition by the angiotensin AT1 receptor antagonist irbesartan. Eur J Pharmacol 2007; 567: 231-239.

3 Mehta PK, Griendling KK. Angiotensin II cell signaling: physiological and pathological effects in the cardiovascular system. Am J Physiol Cell Physiol 2007; 292: C82-C97.

4 Mulvany MJ. Effects of angiotensin-converting enzyme inhibition on vascular remodeling of resistance vessels in hypertensive patients. Metabolism 1998; 47: 20-23.

5 Touyz RM. The role of angiotensin II in regulating vascular structural and functional changes in hypertension. Curr Hypertens Rep 2003; 5: 155-164.

6 Levy Bl. How to explain the differences between renin angiotensin system modulators. Am J Hypertens 2005; 18: 134S-141S.

7 Mulvany MJ. Small artery remodeling and significance in the development of hypertension. News Physiol Sci 2002; 17: 105-109.

8 De Mey JG, Schiffers PM, Hilgers RH, Sanders MM. Toward functional genomics of flow-induced outward remodeling of resistance arteries. Am J Physiol Heart Circ Physiol 2005; 288: H1022-H1027.

9 Lehoux S, Tronc F, Tedgui A. Mechanisms of blood flow-induced vascular enlargement. Biorheology 2002; 39: 319-324.

10 Bakker EN, Buus CL, Spaan JA, Perree J, Ganga A, Rolf TM, Sorop O, Bramsen LH, Mulvany MJ, Vanbavel E. Small artery remodeling depends on tissue-type transglutaminase. Circ Res 2005; 96: 119-126. 
11 Hilgers RH, Schiffers PM, Aartsen WM, Fazzi GE, Smits JF, De Mey JG. Tissue angiotensin-converting enzyme in imposed and physiological flow-related arterial remodeling in mice. Arterioscler Thromb Vasc Biol 2004; 24: 892-897.

12 Matrougui K, Loufrani L, Heymes C, Levy BI, Henrion D. Activation of AT(2) receptors by endogenous angiotensin $\mathrm{II}$ is involved in flow-induced dilation in rat resistance arteries. Hypertension 1999; 34: 659-665.

13 Matrougui K, Levy BI, Henrion D. Tissue angiotensin II and endothelin-1 modulate differently the response to flow in mesenteric resistance arteries of normotensive and spontaneously hypertensive rats. Br J Pharmacol 2000; 130: 521-526.

14 Pinaud F, Bocquet A, Dumont O, Retailleau K, Baufreton C, Andriantsitohaina R, Loufrani L, Henrion D. Paradoxical role of angiotensin II type 2 receptors in resistance arteries of old rats. Hypertension 2007; 50: 96-102.

15 You D, Loufrani L, Baron C, Levy BI, Widdop RE, Henrion D. High blood pressure reduction reverses angiotensin II type 2 receptor-mediated vasoconstriction into vasodilation in spontaneously hypertensive rats. Circulation 2005; 111: 1006-1011.

16 Cousin M, Custaud MA, Baron-Menguy C, Toutain B, Dumont O, Guihot AL, Vessieres E, Subra JF, Henrion D, Loufrani L. Role of angiotensin II in the remodeling induced by a chronic increase in flow in rat mesenteric resistance arteries. Hypertension 2010; 55: 109-115.

17 Dumont O, Loufrani L, Henrion D. Key role of the NO-pathway and matrix metalloprotease- 9 in high blood flow-induced remodeling of rat resistance arteries. Arterioscler Thromb Vasc Biol 2007; 27: 317-324.

18 Loufrani L, Matrougui K, Gorny D, Duriez M, Blanc I, Levy BI, Henrion D. Flow (shear stress)-induced endothelium-dependent dilation is altered in mice lacking the gene encoding for dystrophin. Circulation 2001; 103: 864-870.

19 Lawrenson L, Poole JG, Kim J, Brown C, Patel P, Richardson RS. Vascular and metabolic response to isolated small muscle mass exercise: effect of age. Am J Physiol Heart Circ Physiol 2003; 285: H1023-H1031.

20 Pourageaud F, De Mey JG. Structural properties of rat mesenteric small arteries after 4-wk exposure to elevated or reduced blood flow. Am J Physiol 1997; 273: H1699-H1706.

21 Belin de Chantemele EJ, Vessieres E, Dumont O, Guihot AL, Toutain B, Loufrani L, Henrion D. Reactive oxygen species are necessary for high flow (shear stress)-induced diameter enlargement of rat resistance arteries. Microcirculation 2009; 16: 391-402.

22 Loufrani L, Li Z, Levy BI, Paulin D, Henrion D. Excessive microvascular adaptation to changes in blood flow in mice lacking gene encoding for desmin. Arterioscler Thromb Vasc Biol 2002; 22: 1579-1584.

23 Loufrani L, Levy BI, Henrion D. Defect in microvascular adaptation to chronic changes in blood flow in mice lacking the gene encoding for dystrophin. Circ Res 2002; 91 : 1183-1189.

24 Gorny D, Loufrani L, Kubis N, Levy BI, Henrion D. Chronic hydralazine improves flow (shear stress)-induced endothelium-dependent dilation in mouse mesenteric resistance arteries in vitro. Microvasc Res 2002; 64: 127-134.

25 Dowell FJ, Henrion D, Benessiano J, Poitevin P, Levy B. Chronic infusion of low-dose angiotensin II potentiates the adrenergic response in vivo.J Hypertens 1996; 14: 177-182.

26 Dowell FJ, Henrion D, Duriez M, Michel JB. Vascular reactivity in mesenteric resistance arteries following chronic nitric oxide synthase inhibition in Wistar rats. Br J Pharmacol 1996; 117: 341-346.

27 Henrion D, Laher I, Bevan JA. Intraluminal flow increases vascular tone and 45Ca2+ influx in the rabbit facial vein. Circ Res 1992; 71: 339-345.

28 Iglarz M, Matrougui K, Levy BI, Henrion D. Chronic blockade of endothelin ETA receptors improves flow dependent dilation in resistance arteries of hypertensive rats. Cardiovasc Res 1998; 39: 657-664.
29 Belin de Chantemele EJ, Retailleau K, Pinaud F, Vessieres E, Bocquet A, Guihot AL, Lemaire B, Domenga V, Baufreton C, Loufrani L, Joutel A, Henrion D. Notch3 is a major regulator of vascular tone in cerebral and tail resistance arteries. Arterioscler Thromb Vasc Biol 2008; 28: 2216-2224.

30 Loufrani L, Dubroca C, You D, Li Z, Levy B, Paulin D, Henrion D. Absence of dystrophin in mice reduces NO-dependent vascular function and vascular density: total recovery after a treatment with the aminoglycoside gentamicin. Arterioscler Thromb Vasc Biol 2004; 24: 671-676.

31 Mulvany MJ, Halpern W. Contractile properties of small arterial resistance vessels in spontaneously hypertensive and normotensive rats. Circ Res 1977; 41: 19-26.

32 Bouvet $C$, Belin de Chantemele E, Guihot AL, Vessieres E, Bocquet A, Dumont O, Jardel A, Loufrani L, Moreau P, Henrion D. Flow-induced remodeling in resistance arteries from obese Zucker rats is associated with endothelial dysfunction. Hypertension 2007; 50: 248-254.

33 Dumont O, Pinaud F, Guihot AL, Baufreton C, Loufrani L, Henrion D. Alteration in flow (shear stress)-induced remodelling in rat resistance arteries with aging: improvement by a treatment with hydralazine. Cardiovasc Res 2008; 77: 600-608.

34 Loufrani L, Retailleau K, Bocquet A, Dumont O, Danker K, Louis H, Lacolley P, Henrion D. Key role of alpha(1)beta(1)-integrin in the activation of PI3-kinase-Akt by flow (shear stress) in resistance arteries. Am J Physiol Heart Circ Physiol 2008; 294: H1906-H1913.

35 Bagi Z, Toth E, Koller A, Kaley G. Microvascular dysfunction after transient high glucose is caused by superoxide-dependent reduction in the bioavailability of $\mathrm{NO}$ and $\mathrm{BH}(4)$. Am J Physiol Heart Circ Physiol 2004; 287: H626-H633.

36 Belin de Chantemele EJ, Vessieres E, Guihot AL, Toutain B, Loufrani L, Henrion D. Cyclooxygenase-2 preserves flow-mediated remodelling in old obese Zucker rat mesenteric arteries. Cardiovasc Res.

37 Pourageaud F, De Mey JG. Vasomotor responses in chronically hyperperfused and hypoperfused rat mesenteric arteries. Am J Physiol 1998; 274: H1301-H1307.

38 Higashi Y, Chayama K, Yoshizumi M. Angiotensin II type I receptor blocker and endothelial function in humans: role of nitric oxide and oxidative stress. Curr Med Chem Cardiovasc Hematol Agents 2005; 3: 133-148.

39 Touyz RM. Reactive oxygen species as mediators of calcium signaling by angiotensin II: implications in vascular physiology and pathophysiology. Antioxid Redox Signal 2005; 7: $1302-1314$.

40 Bakker EN, Matlung HL, Bonta P, de Vries CJ, van Rooijen N, Vanbavel E. Blood flowdependent arterial remodelling is facilitated by inflammation but directed by vascular tone. Cardiovasc Res 2008; 78: 341-348.

41 Bakker EN, van der Meulen ET, van den Berg BM, Everts V, Spaan JA, VanBavel E. Inward remodeling follows chronic vasoconstriction in isolated resistance arteries. J Vasc Res 2002; 39: 12-20.

42 Henrion D, Dowell FJ, Levy BI, Michel JB. In vitro alteration of aortic vascular reactivity in hypertension induced by chronic NG-nitro-L-arginine methyl ester. Hypertension 1996; 28: 361-366.

43 Qiu HY, Henrion D, Levy BI. Alterations in flow-dependent vasomotor tone in spontaneously hypertensive rats. Hypertension 1994; 24: 474-479.

44 Mulvany MJ. Abnormalities of the resistance vasculature in hypertension: correction by vasodilator therapy. Pharmacol Rep 2005; 57(Suppl): 144-150.

45 Khavandi K, Greenstein AS, Sonoyama K, Withers S, Price A, Malik RA, Heagerty AM. Myogenic tone and small artery remodelling: insight into diabetic nephropathy. Nephrol Dial Transplant 2009; 24: 361-369.

46 Drexler H, Hornig B. Endothelial dysfunction in human disease. J Mol Cell Cardiol 1999; 31: 51-60.

Supplementary Information accompanies the paper on Hypertension Research website (http://www.nature.com/hr) 